This is the post peer-review accepted manuscript of:

B. Milosevic, E. Farella and S. Benatti, "Exploring Arm Posture and Temporal Variability in Myoelectric Hand Gesture Recognition," 2018 7th IEEE International Conference on Biomedical Robotics and Biomechatronics (Biorob), Enschede, 2018, pp. 1032-1037.

The published version is available online at:

https://doi.org/10.1109/BIOROB.2018.8487838

(C) 2018 IEEE. Personal use of this material is permitted. Permission from IEEE must be obtained for all other uses, in any current or future media, including reprinting/republishing this material for advertising or promotional purposes, creating new collective works, for resale or redistribution to servers or lists, or reuse of any copyrighted component of this work in other works. 


\title{
Exploring Arm Posture and Temporal Variability in Myoelectric Hand Gesture Recognition
}

\author{
Bojan Milosevic ${ }^{1}$, Elisabetta Farella ${ }^{1}$ and Simone Benatti ${ }^{2}$
}

\begin{abstract}
Hand gesture recognition based on myoelectric (EMG) signals is an innovative approach for the development of intuitive interaction devices, ranging from poliarticulated prosthetic hands to intuitive robot and mobile interfaces. Their study and development in controlled environments provides promising results, but effective real-world adoption is still limited due to reliability problems, such as motion artifacts and arm posture, temporal variability and issues caused by the re-positioning of sensors at each use. In this work, we present an EMG dataset collected with the aim to explore postural and temporal variability in the recognition of arm gestures. Its collection of gestures executed in $\mathbf{4}$ arm postures over 8 days allows to evaluate the impact of such variability on classification performance. We implemented and tested Stateof-the-Art (SoA) recognition approaches analyzing the impact of different training strategies. Moreover, we compared the computational and memory requirements of the considered algorithms, providing an additional evaluation criteria useful for real-time implementation. Results show a decrease in the recognition of inter-posture and inter-day gestures up to $20 \%$. The provided dataset will allow further exploration of such effects and the development of effective training and recognition strategies.
\end{abstract}

\section{INTRODUCTION}

The Electromyogram (EMG) is the biopotential signal resulting from muscular activity, it can be sensed using noninvasive surface electrodes and processed to implement myoelectric Human-Machine Interfaces (HMIs). Recent research highlights its potential to enable new interaction paradigms in applications such as natural prosthetic control [1], robot interaction [2], game or mobile interfaces [3]. Hand gesture recognition based on forearm EMG signals is an enabling technology for the development of advanced and intuitive interaction strategies [4]. Its application to the development of naturally-controlled poliarticulated hand prosthesis is an interesting research challenge with promising results and the potential to dramatically improve the quality of life for forearm amputees [5], [6].

Additional application scenarios include HMI for industrial robot control [7], [8], interactions for virtual environments [9] or rehabilitation [10]. Industrial interest and the availability of first commercial solutions taking advantage of EMG-based interaction highlight the potential of this approach and its wider use [11], [12].

Several studies have proven recognition capabilities in controlled environments, but effective real-world adoption of EMG-based HMIs is still limited due to reliability

\footnotetext{
${ }^{1}$ E3DA, ICT Center, FBK, Trento, Italy;

email: \{milosevic, efarella\}efbk.eu

${ }^{2}$ Micrel Lab, Unversity of Bologna, Italy;

email: simone.benatti@unibo.it
}

problems [11], [13]. Research efforts now focus on more complex evaluations including postural effects and long term reliability [14], however the efforts of the research community are still fragmented and suffer from the lack of common benchmarks and datasets suitable for the analysis of variability.

In this work, we present a dataset, which aims to explore the effects of arm posture and temporal variability in myoelectric hand gesture recognition. Building on the preliminary analysis in [15], the dataset includes 7 subjects performing 6 discrete gestures in 4 arm postures and repeated for 8 days, totaling 224 independent sessions. The acquisition is based on 4 commercial bipolar electrodes interfaced with an embedded 16-bit ADC, providing a repeatable setup suitable for integrated prosthetic controllers [16]. Its composition and acquisition setup make it the first EMG dataset to date to include both postural and temporal variability and the public availability ${ }^{1}$ will help further research efforts in the development of reliable myoelectric HMIs.

With the collected data, we analyze SoA recognition approaches, focusing on the impact of the composition of training and testing data. In particular, we analyze intra(training and test from same posture/day) and inter-(training and test from different postures/days) combinations, which highlight the impact of such variability on the different algorithms. Results show a classification accuracy higher than $90 \%$ for intra-posture and day analysis, which suffers a degradation of up to $20 \%$ when testing on data from different postures or days, in accordance with existing literature [17], [18]. Therefore, in this paper we demonstrate that accuracy decline is mitigated by using combinations of data from multiple sessions to train the recognition algorithms and the presented dataset provides a unique opportunity to further develop effective training strategies.

As an additional contribution, we compare the selected recognition algorithms in terms of computation and memory needs, considering both training and prediction requirements. We observe that different algorithms with different computational loads are similarly affected by data variability and similarly improves their performance with the training set. This is of paramount importance when selecting an algorithm for a real-time implementation targeting embedded and resource-constrained platforms.

\section{RELATED WORK}

The influence of arm posture during the execution of gestures is an important effect to be addressed when evaluating

${ }^{1}$ github.com/simonebenatti/EMG_UNIBO_FBK_DATASET 


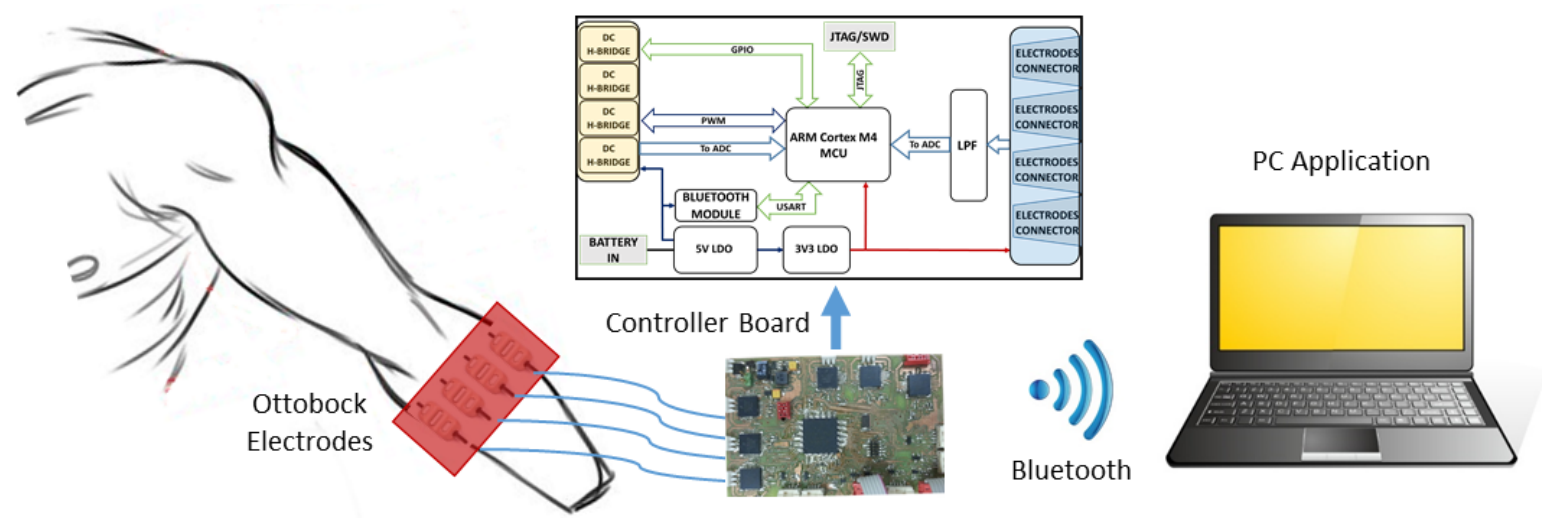

Fig. 1. Data acquisition setup and the architectural diagram of the acquisition board.

recognition strategies. The work in [17] evaluates feature extraction approaches to classify 8 discrete gestures in 5 arm postures, while [19] evaluates a regression technique to proportionally estimate wrist movements in 6 arm postures. The recognition of 6 different gestures in 3 arm orientations is analyzed in [20] and all the three works report similar findings with recognition accuracy above $90 \%$ when training and testing with data from the same postures. However, performance declines of up to $20 \%$ when training and testing are performed on data from different postures.

Long term use and temporal reliability are further effects to be addressed, including issues such as fatigue, user adaptation and the variability introduced from the re-positioning of electrodes at each data collection session. In [18], one subject performed 10 discrete gestures over a period of 21 days, for a total of 121 sessions and such data was used to test commonly adopted algorithms when trained on different combinations of preceding sessions. Results show that training on the first 5 sessions caused mean errors higher than $20 \%$, while training on the latest 5 or all previous sessions lowered the mean error at 10-12\%. In [21], 5 subjects performed 8 gestures over 5 days and intra-day accuracy was evaluated by random 5-fold cross validation, while inter-day accuracy was evaluated training with a whole day's session and testing on all the others. In this case, the authors observed that the classification performance decreased monotonically on average by $4.1 \%$ a day. User adaptation in the execution of 13 gestures over 11 days was studied in [22]. The authors evaluated between-day performance training on one day at a time and testing on the next day and on the 3rd day. The results show that in both cases the error is higher for the first days and then decreases, indicating that the differences in gesture execution decrease over time.

The above works provide interesting results on the effects of postural or temporal variability, however they do not provide the collected data and hence limit the research efforts towards improvement. Significant contribution in this direction was provided by the Ninapro database [23], which collects acquisitions totaling 67 subjects performing up to 52 hand movements. It focuses on gesture and subject variability collecting single day sessions, but the authors recently published an extension with data from 10 subjects performing 7 gestures over 5 days, with 2 sessions per day [24]. The recognition of afternoon sessions when training on morning data shows a decrease in accuracy of $27 \%$. The dataset presented in this work provides the widest variability to date, including both postural and temporal evaluations with a total of 224 independent sessions. Such features allows us to study the performance of classification approaches in a more realistic scenario and to evaluate training approaches capable to improve the recognition accuracy.

\section{MAterials AND Methods}

\section{A. EMG signal}

The EMG signal is a major index of the muscular activity. The depolarization of the tissue cell membrane, caused by the $\mathrm{Na}^{+}$and $\mathrm{K}^{-}$ions flow, propagates along the fibers during a muscular contraction. Such stimulus starts from the central nervous system and goes through the motoneurons that are innervated in the muscular tissue. These potentials are named Action Potentials (APs) and they can be detected by an instrumentation amplifier with the positive and negative terminals each one connected to 2 metal plates placed on the skin surface. The superposition of all the underlying APs detected by the amplifier forms the EMG signal.

\section{B. Acquisition Setup}

The EMG signal amplitude ranges from $10 \mu \mathrm{V}$ to $10 \mathrm{mV}$, depending on size and distance of the muscles underlying the electrodes, while the signal bandwidth stays within $2 k H z$. Several noise sources affect such signals, e.g. motion artifacts, floating ground noise, crosstalk and Power Line Interference (PLI) [25]. In order to provide a reliable and repeatable setup, we used a commercial sensor, the Ottobock 13E200 pre-amplified single-ended EMG electrode. It amplifies and integrates the raw EMG signal to reach an output span of $0-3.3 \mathrm{~V}$, suitable for the single-ended interface of the ADC of an embedded microcontroller. The sensors integrate an analog notch filter to remove the PLI noise, while their bandwidth spans between 90 and $450 \mathrm{~Hz}$. The output analog signals were acquired with a custom embedded board based on an NXP K40 ARM Cortex M4 microcontroller equipped with an internal 16-bit SAR ADC [26]. The architectural diagram of the board is shown in Fig.1. Digitalized signals 

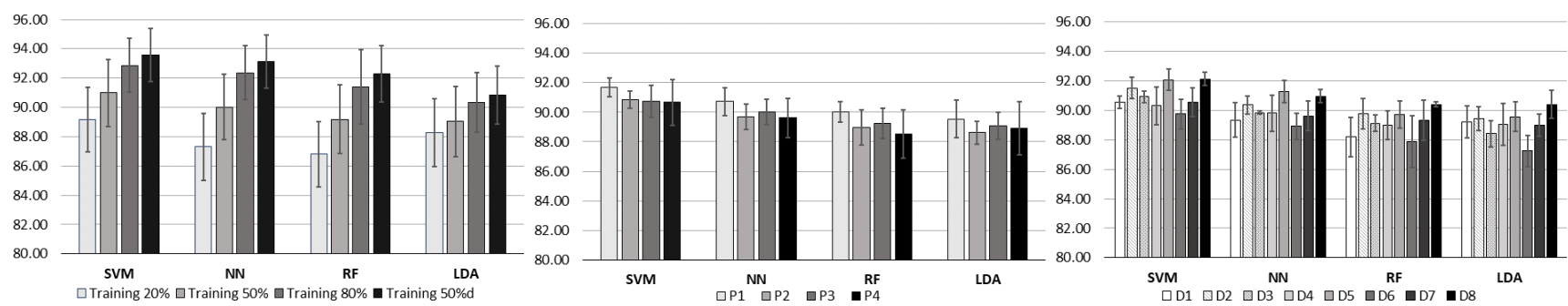

Fig. 2. Classification rates when training and testing using different splits from the same session, averaged across all subjects. Left: average for all data; Center: average for the different arm postures; Right: average for the different days.

were then streamed via Bluetooth to a laptop where they were stored and used for offline data analysis performed in Matlab.

\section{Experimental protocol}

For the dataset collection we selected common hand gestures used in daily life, i.e. power grip, 2 fingers pinch grip, 3 fingers pinch grip, pointing index and open hand. We also included the rest position, recorded when muscles are relaxed between 2 subsequent movement repetitions. During the experiment, the subjects worn an elastic armband with 4 Ottobock sensors placed on the forearm muscles (Fig. 3) involved in the selected movements (i.e. extensor carpi ulnaris, extensor comunis digitorum, flexor carpi radialis and flexor carpi ulnaris). Sensors were placed on the proximal third at $30 \mathrm{~mm}$ respectively on the left and on the right side of 2 axial lines ideally traced on the forearm. No markings were used for the positioning of the sensors, but for each session they were carefully placed at the same position on the subject's arm.

The data collection includes 7 healthy subjects (all male, $29.5 \pm 12.2$ years) with gestures acquired in 4 sessions per day, one for each posture, over 8 days totaling 224 independent sessions. The study was approved by the local ethics committee and the subjects signed an informed consent prior to their participation. Each session contains 10 repetitions of each gesture, with 3 second contractions interleaved by 3 seconds of muscular relaxation (i.e. the rest gesture). The collected arm postures are: proximal (P1), distal (P2), distal with the palm oriented down (P3) and distal with the arm lifted up by 45 degrees (P4). The posture and gesture sequences were randomized at each session. At the

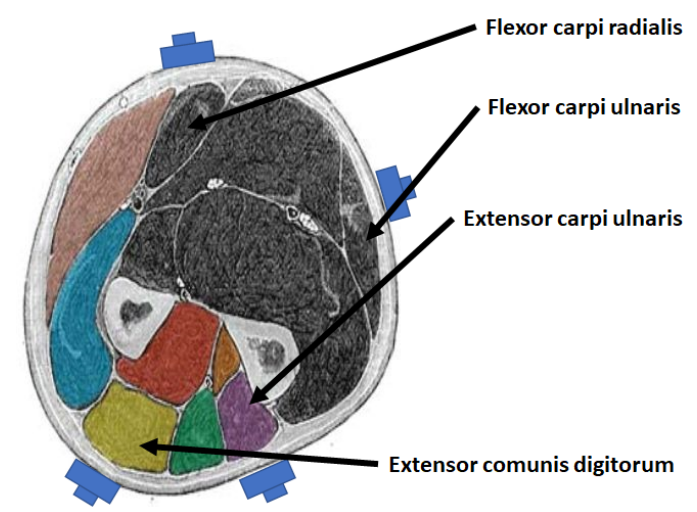

Fig. 3. Forearm muscle structure and sensors placement. end of a session, each trace has been visually inspected to check the quality of the acquisition. Gesture segmentation was performed with a combination of manual inspection and an adaptive threshold to separate contractions from rest. The dataset is made publicly available with a series of Matlab scripts, to help users in its analysis.

\section{Data Analysis}

Several machine learning algorithms, with varying approaches regarding signal acquisition, pre-processing and feature extraction have been successfully applied to EMG gesture recognition [4]. In this work, we aim to exploit the postural and temporal variability represented in the dataset to analyze the performance and for well established recognition approaches. Such analysis allows us to get a step out of the lab and closer to the complexity of real-life scenarios. The algorithms we choose to compare are: Support Vector Machine (SVM), Neural Networks (NN), Random Forest (RF) and Linear Discriminant Analysis (LDA). Recently, also deep learning techniques have been used with promising results when applied to large datasets and multidimensional inputs [27]. However, with a more limited setup such as the one used here, the improvement over standard techniques is limited [28]. This work does not include the deep learning approaches, which will be considered for future work, as we want to focus on the impact of different training strategies and on the comparison of the most used and robust approaches for EMG classification.

We use a low-pass filter as the pre-processing stage and we feed such filtered signals as input for the recognition algorithms without further feature extraction. Such choice allows us to provide a common processing pipeline for all the considered algorithms and was based on preliminary analysis and literature results showing good performance of similar features such as the mean absolute value [23], [24].

A preliminary analysis was also used to optimize the parameters of each algorithm. In particular, we used a subset of 32 randomly selected sessions and performed a grid search for the main algorithm variants and their parameters. The resulting best configuration was used for the rest of the analysis as follows: filter: low-pass exponential IIR filter with $\alpha=0.01$; SVM: RBF kernel with $C=1$; NN: twolayer fully connected feed-forward network with 8 sigmoid hidden and 6 softmax output neurons; RF: 40 trees with 2 randomly chosen features; LDA: $\delta=0, \gamma=0$. For all the performed evaluations, we use the mean of 5 runs, with randomly initialized parameters. 

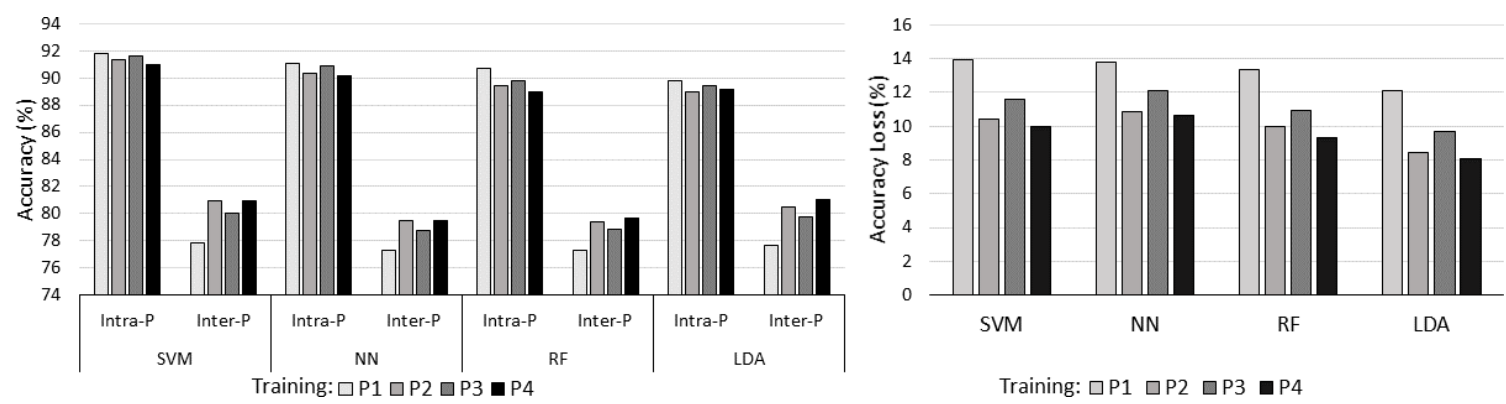

Fig. 4. (Left) Average recognition accuracy when training with data from a single posture and testing on itself (intra-P) or on the others (inter-P). (Right) Average accuracy loss between intra-P and inter-P accuracy.
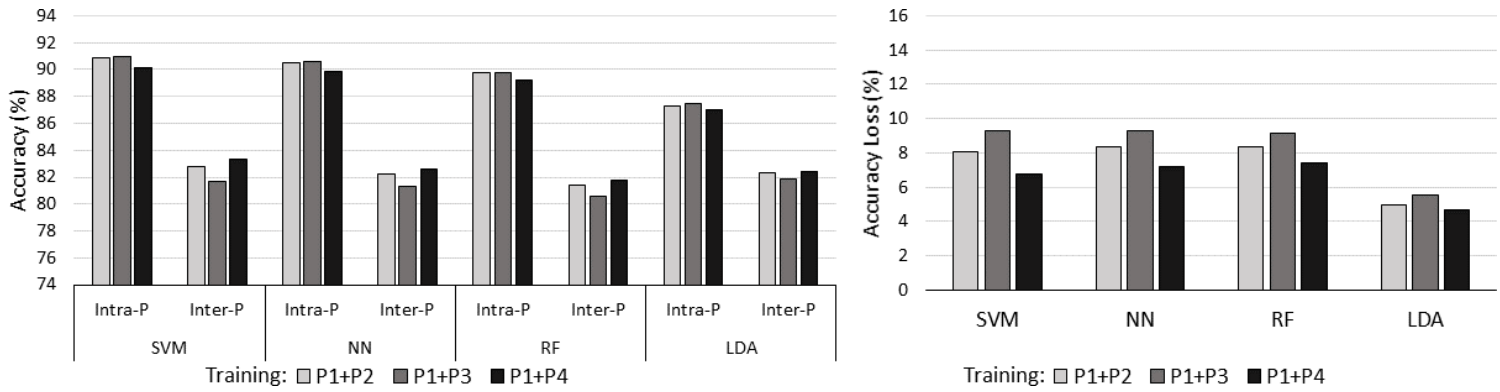

Fig. 5. (Left) Average recognition accuracy when training with data from a 2 postures and testing on the same ones (intra-P) or on the others (inter-P). (Right) Average accuracy loss between intra-P and inter-P accuracy.

\section{Results}

\section{A. Single session training and recognition}

To provide a reference performance, we used each individual session to train and test the recognition algorithms and evaluate their accuracy within the different postures/days. Available studies use various methods to partition training and test data, even in single-session analysis. Alternatives include 5-fold cross validation [21] or using some of the first contractions [17], [20] or using contractions spaced throughout the session, simulating a more equally distributed division [23], [24]. In order to evaluate their effects, we performed the following train/test splits on each session's data: Training 20\% (5-fold linear split alternating 1 fold for training and the rest for test), Training 50\% (2-fold linear split, alternating them for training/test), Training $80 \%$ (5-fold linear split alternating 4 folds for training and the remaining for test), Training 50\%D (10-fold linear split, alternating the odd and the even ones for training/test). All the splits were performed preserving the integrity of gesture contractions: with 10 repetitions per gesture, each contraction was assumed to represent $10 \%$ of the session data.

The mean classification accuracy over all the available sessions is reported in Fig. 2 (left) and we can note how the four considered algorithms have a very close performance. Moreover, the amount and distribution of training data has a notable influence, specially for NN and RF. Overall, the SVM has a slightly better performance, while the LDA is the one less subject to the influence of training data selection.

In addition to the average over all the sessions, we also grouped the results by postures and by days. Such analysis is reported in Fig. 2 (center) for the different postures and in Fig. 2 (right) for the different days. In both cases, here we report the Training 50\% split as an example, while the other ones had similar results. Regarding the different postures, we can see an almost equal performance, with the mean differences within $2 \%$ for each algorithm. The performance on the different days has a major variability, but there is not a defined pattern or trend among the days.

\section{B. Inter-posture recognition}

To evaluate inter-posture recognition capabilities, we trained the different algorithms on data from a given posture and tested them on all the others. In this case, we used the first $50 \%$ of data to train each posture's models and applied it to the other $50 \%$ to evaluate the intra-posture accuracy or to all data from the other postures of the same day for interposture accuracy. We used a contiguous split of data at the beginning of a session to simulate a user-performed training. Different amounts of training data did not produce significant differences, except an overall shift in accuracy aligned with the single-session case.

The recognition accuracy is reported in Fig. 4 (left) and the decrease in recognition for out-of-posture gestures is reported in Fig. 4 (right). SVM has the highest absolute accuracy, but the LDA has the lower out-of-posture degradation. Moreover, using P1 for training consistently leads to higher accuracy loss when compared to the other postures. While P1 is the most commonly used posture in EMG literature, it differs from P2-P4 that all have the arm fully extended and hence have more homogeneous results. Moreover, P3 has a different wrist orientation, which introduces additional difference compared to $\mathrm{P} 2$ and $\mathrm{P} 4$.

In addition to single-posture training, we also tested the ability of the algorithms to better generalize when trained on data from 2 postures. In this case, we again used 50\% 


\section{Training:}
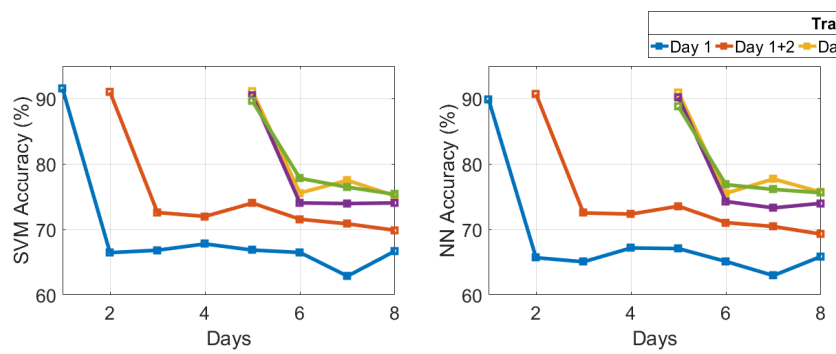

Fig. 6. Average accuracy when training on different combinations of days and testing on

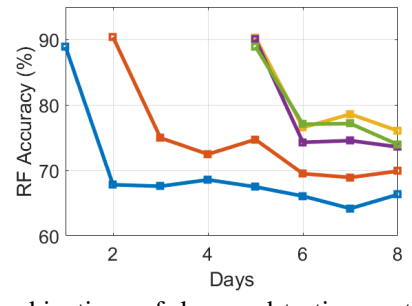

F. 5. In this case, on the trained postures we have loss of accuracy when compared to the single posture case, but the out-of-posture recognition improves with a smaller accuracy loss.

\section{Inter-day recognition}

Given the variety of the collected dataset, numerous temporal train/test combinations can be analyzed. We aim to simulate user-performed training, hence we enforce a temporal progression and we restrict the inter-day tests only on days following the training one(s). For the same reason, we use the first $50 \%$ of data from a given day for training and test on the remaining data to evaluate intra-day accuracy or on data from the following days. The analyzed training combinations include: single day, the first two days, the last two days and all the previous days. Figure 6 summarizes the results, where for clarity we plot a subset of combinations with training on days 1 to 5 and testing on the last 3 days.

All the evaluated algorithms show a marked decline of up to $20 \%$ in inter-day recognition accuracy when trained with data from a single day. Using data from the first 2 days (D1+D2) improves the performance, but with a limited effect on the later days. More effective improvement is provided when including data from the latest days. Using the last two days (D4+D5) provides the best results, while using all the previous days (D1 to D5) does not add significant advantage. Such results also show an user adaptation effect: training with data from the last available 2 days provides better results for the later days (e.g. training D4+D5, test D6), when compared to the earlier ones (e.g. training D1+D2, test D3).

\section{Computational and memory requirements}

An effective real-world deployment should also consider the computational and memory requirements of a recognition approach and the computing platforms needed for its implementation. However, such aspects are usually ignored when providing evaluations of EMG-based recognition strategies. Despite the offline approach adopted for our analysis, we wanted to evaluate and compare the selected algorithms also from a computational perspective, to add further insights in their performance.

In this work, all the analysis of the recognition capabilities was performed offline and implemented in Matlab. In addition to the recognition results, we logged the times needed for the training/recognition tasks and the memory requirements for the resulting models. We used the implementations of
TABLE

COMPUTATIONAL AND MEMORY REQUIREMENTS FOR THE TESTED ALGORITHMS. AVERAGE RESULTS WITH 50\% TRAINING DATA FROM

EACH CONSIDERED SESSION.

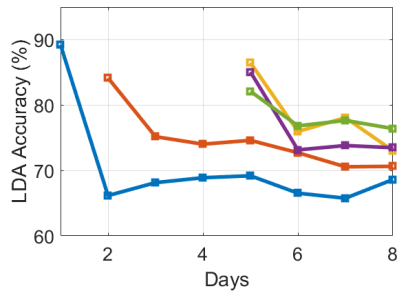

the subsequent days.

\begin{tabular}{lrrrr}
\hline \hline Train time per session $(s)$ & SVM & NN & RF & LDA \\
\hline Training 1 Session & 23.39 & 16.09 & 15.33 & 0.11 \\
Training 2 Postures & 96.90 & 41.45 & 36.07 & 0.22 \\
Training 2 Days & 81.30 & 40.28 & 35.16 & 0.26 \\
Training 4 Days & 412.63 & 119.01 & 79.79 & 0.43 \\
\hline \hline Predict time per sample $(\mu s)$ & & & & \\
\hline Training 1 Session & 254.00 & 0.60 & 27.10 & 1.01 \\
Training 2 Postures & 735.00 & 0.53 & 30.60 & 1.04 \\
Training 2 Days & 534.04 & 0.55 & 33.69 & 1.04 \\
Training 4 Days & 1724.71 & 0.49 & 31.29 & 1.02 \\
\hline \hline Memory Occupation $(k B)$ & & & & \\
\hline Training 1 Session & 830 & 39 & 10996 & 26 \\
Training 2 Postures & 2186 & 39 & 28400 & 26 \\
Training 2 Days & 1557 & 39 & 19009 & 26 \\
Training 4 Days & 4884 & 39 & 60327 & 26 \\
\hline \hline
\end{tabular}

the different algorithms as provided by the Matlab's native Statistical and Neural Network Toolboxes (ver. Matlab R2016b) and all the processing was performed on a PC (dual core 64 bit CPU Intel i5-4310U, 2.60GHz, 8Gb RAM, with Windows 1064 bit). For the training times, we consider the time needed to train each model, regardless of the exact size of the training data set. For the prediction times, we still measured the time needed to process the whole test set and we normalized the results dividing by the number of samples to have an estimate of the processing time per sample. The average results for this analysis are collected in Table I. The computational evaluation is highly dependant on the exact implementation and computing architecture used, hence the absolute results may be of little general interest. However, having a consistent setup and using the same implementation and computing platform for all the algorithms, their relative comparison is of general validity. Regarding training and prediction times, our results show that the SVM has up to 3 orders of magnitude higher requirements when compared to the other algorithms and the LDA exhibits the lowest needs. Moreover, NN and LDA have constant prediction times, while for SVM and RF they depend on the complexity of the trained models. The same holds for memory requirements, where again NN and LDA have constant and considerably 
smaller memory footprints.

For all the algorithms the training times increase with the increase of the training data set, but for the SVM this effect has the most evident impact. With larger and more complex training data, the SVM model needs more support vectors to effectively separate the different classes and this impacts directly the resource needs. Similarly, the RF suffers from very high memory requirements increasing with the complexity of the model, but its computational requirements remain limited. NN and LDA, exhibit the best performance, with very limited and constant prediction and memory requirements driven by the nature of such algorithms, which have fixed models.

\section{CONCLUSION}

In this work, we presented a dataset for the evaluation of arm posture and temporal variability in myoelectric hand gesture recognition. The results can be used as guidelines for the development of training and recognition approaches for gesture-based EMG HMIs. In particular, it is clear that multi-posture and multi-day training strategies are needed to improve the performance of the classification algorithms. The evaluated algorithms have similar recognition performance with the SVM providing the highest accuracy and the LDA providing the lowest accuracy losses for inter-posture and day analysis. Moreover, results on temporal variability show a progressive user adaptation trend and indicate that (re)training strategies should prioritize the availability of recent data. From a computational resources perspective, the algorithms exhibit important differences, with the SVM having up to 3 orders of magnitude higher needs for both training and prediction tasks. Even if the reported results can be drastically improved by the optimization of the algorithms implementations, their comparison offers useful insights to weight the recognition results when targeting realtime implementations. This work conducted a preliminary analysis on the collected dataset, which unique postural and temporal variability allows for further development of robust recognition approaches. The public release of such data aims to provide a starting point for the research community to address reliability issues and advance myoelectric HMIs towards effective adoption.

\section{ACKNOWLEDGMENTS}

The research contribution presented in this paper hasbeen supported by the European project EuroCPS (grant n.644090).

\section{REFERENCES}

[1] D. Farina et al., "The extraction of neural information from the surface emg for the control of upper-limb prostheses: emerging avenues and challenges," IEEE Trans. on Neural Syst. and Rehab. Eng., vol. 22 (4), pp. 797-809, 2014.

[2] R. Meattini et al., "An sEMG-based human-robot interface for robotic hands using machine learning and synergies," IEEE Transactions on Components, Packaging and Manufacturing Technology, 2018.

[3] T. S. Saponas et al., "Making muscle-computer interfaces more practical," in SIGCHI Conf. on Human Factors in Computing Systems, 2010, pp. 851-854.
[4] M. Hakonen et al., "Current state of digital signal processing in myoelectric interfaces and related applications," Biomedical Signal Processing and Control, vol. 18, pp. 334-359, 2015.

[5] J. L. Segil et al., "Comparative study of state-of-the-art myoelectric controllers for multigrasp prosthetic hands," Journal of rehabilitation research and development, vol. 51, no. 9, p. 1439, 2014.

[6] I. Kyranou, A. Krasoulis, M. S. Erden, K. Nazarpour, and S. Vijayakumar, "Real-time classification of multi-modal sensory data for prosthetic hand control," in IEEE Intl. Conf. on Robotics and Biomimetics (ROBIO). IEEE, 2016, pp. 536-541.

[7] D. R. Freer et al., "Optimization of emg movement recognition for use in an upper limb wearable robot," in Wearable and Implantable Body Sensor Networks (BSN), 2017, pp. 202-205.

[8] R. Meattini, S. Benatti, U. Scarcia, L. Benini, and C. Melchiorri, "Experimental evaluation of a semg-based human-robot interface for human-like grasping tasks," in IEEE Intl. Conf. on Robotics and Biomimetics (ROBIO). IEEE, 2015, pp. 1030-1035.

[9] S. Gieser, V. Kanal, and F. Makedon, "Evaluation of a low cost emg sensor as a modality for use in virtual reality applications," in Intl. Conf. on Virtual, Augmented and Mixed Reality. Springer, 2017, pp. 97-110.

[10] A. Darzi, M. Goršič, and D. Novak, "Difficulty adaptation in a competitive arm rehabilitation game using real-time control of arm electromyogram and respiration," in Intl. Conf. on Rehabilitation Robotics (ICORR). IEEE, 2017, pp. 857-862.

[11] M. Atzori et al., "Control capabilities of myoelectric robotic prostheses by hand amputees: a scientific research and market overview," Frontiers in systems neuroscience, vol. 9, 2015.

[12] I. Donovan et al., "Myohmi: A low-cost and flexible platform for developing real-time human machine interface for myoelectric controlled applications," in IEEE Intl. Conf. on Systems, Man, and Cybernetics (SMC). IEEE, 2016, pp. 4495-4500.

[13] B. Milosevic, S. Benatti, and E. Farella, "Design challenges for wearable emg applications," in Design, Automation \& Test in Europe Conference \& Exhibition (DATE). IEEE, 2017, pp. 1432-1437.

[14] E. J. Earley et al., "The effect of wrist position and hand-grasp pattern on virtual prosthesis task performance," in IEEE Intl. Conf. on Robotics and Biomimetics (ROBIO). IEEE, 2016.

[15] S. Benatti et al., "Analysis of robust implementation of an emg pattern recognition based control," in BIOSIGNALS, 2014, pp. 45-54.

[16] Benatti et al., "A prosthetic hand body area controller based on efficient pattern recognition control strategies," Sensors, 2017.

[17] R. Khushaba et al., "Towards limb position invariant myoelectric pattern recognition using time-dependent spectral features," Neural Networks, vol. 55, pp. 42-58, 2014.

[18] P. Kaufmann et al., "Fluctuating emg signals: Investigating long-term effects of pattern matching algorithms," in EMBC, 2010.

[19] K.-H. Park et al., "Position-independent decoding of movement intention for proportional myoelectric interfaces," IEEE Trans. on Neural Sys. and Rehab. Eng., vol. 24 (9), pp. 928-939, 2016.

[20] R. Khushaba and thers, "Combined influence of forearm orientation and muscular contraction on emg pattern recognition," Expert Systems with Applications, vol. 61, pp. 154-161, 2016.

[21] S. Amsuss et al., "Long term stability of surface emg pattern classification for prosthetic control," in EMBC, 2013, pp. 3622-3625.

[22] J. He et al., "User adaptation in long-term, open-loop myoelectric training: implications for emg pattern recognition in prosthesis control," Journal of neural engineering, vol. 12 (4), p. 046005, 2015.

[23] M. Atzori et al., "Characterization of a benchmark database for myoelectric movement classification," IEEE Trans. on Neural Syst. and Rehab. Engineering, vol. 23 (1), pp. 73-83, 2015.

[24] F. Palermo et al., "Repeatability of grasp recognition for robotic hand prosthesis control based on semg data," in Rehabilitation Robotics (ICORR), 2017, pp. 1154-1159.

[25] M. Tomasini et al., "Power line interference removal for high-quality continuous biosignal monitoring with low-power wearable devices," IEEE Sensors Journal, vol. 16, no. 10, pp. 3887-3895, 2016.

[26] "K40," https://www.nxp.com/docs/en/data-sheet/K40P81M100SF2V2. pdf, accessed 20 June 2018.

[27] Y. Du et al., "Surface EMG-based inter-session gesture recognition enhanced by deep domain adaptation," Sensors, 2017.

[28] M. Atzori, M. Cognolato, and H. Müller, "Deep learning with convolutional neural networks applied to electromyography data: a resource for the classification of movements for prosthetic hands," Frontiers in neurorobotics, vol. 10, p. 9, 2016. 